\title{
An Interactive LiDAR to Camera Calibration
}

\author{
Yecheng Lyu, Lin Bai, Mahdi Elhousni and Xinming Huang
}

\begin{abstract}
Recent progress in the automated driving system (ADS) and advanced driver assistant system (ADAS) has shown that the combined use of $3 D$ light detection and ranging (LiDAR) and the camera is essential for an intelligent vehicle to perceive and understand its surroundings. LiDAR-camera fusion requires precise intrinsic and extrinsic calibrations between the sensors. However, due to the limitation of the calibration equipment and susceptibility to noise, algorithms in existing methods tend to fail in finding LiDAR-camera correspondences in long-range. In this paper, we introduced an interactive LiDAR to camera calibration toolbox to estimate the intrinsic and extrinsic transforms. This toolbox automatically detects the corner of a planer board from a sequence of LiDAR frames and provides a convenient user interface for annotating the corresponding pixels on camera frames. Since the toolbox only detects the top corner of the board, there is no need to prepare a precise polygon planar board or a checkerboard with different reflectivity areas as in the existing methods. Furthermore, the toolbox uses genetic algorithms to estimate the transforms and supports multiple camera models such as the pinhole camera model and the fisheye camera model. Experiments using Velodyne VLP-16 LiDAR and Point Grey Chameleon 3 camera show robust results.
\end{abstract}

\section{INTRODUCTION}

Automated driving systems (ADS) and advanced driver assistant systems (ADAS) equipped on intelligent vehicles rely on multiple sensors to perceive their surroundings. In recent research works, LiDAR-based algorithms have shown their advantage on drivable region segmentation [8] [9], object detection [18], and simultaneous localization and mapping [19] [15]. LIDARs are also fused with cameras to improve the accuracy of 3D object detection [2]. However, calibration between LiDAR and camera devices is required to assign the detections to the same coordinate frames so that we can fuse the sensor data. Owing to the development of Multiple View Geometry and computer vision, the models of $3 \mathrm{D}$ to $2 \mathrm{D}$ projection has been well established. Nevertheless, limitations are observed in existing calibration algorithms when applied to LiDAR-camera systems on intelligent vehicles.

Figure 1 shows our autonomous vehicle prototype. The LiDAR-camera system is designed to detect up to 100 meters in front of the vehicle. But most of the existing LiDAR-tocamera calibration algorithms are proposed for indoor use and are validated in meters rage [3] [12] [11]. As shown in Figure 2, the increase of sensing range leads to lower resolution of the camera lens system and larger offset on

This work is partially supported by U.S. NSF Grant CNS-1626236.

All authors are with Department of Electrical and Computer Engineering, Worcester Polytechnic Institute, Worcester, MA 01609, USA. Email: \{ylyu,lbai2, melhousni, xhuang\}@wpi.edu

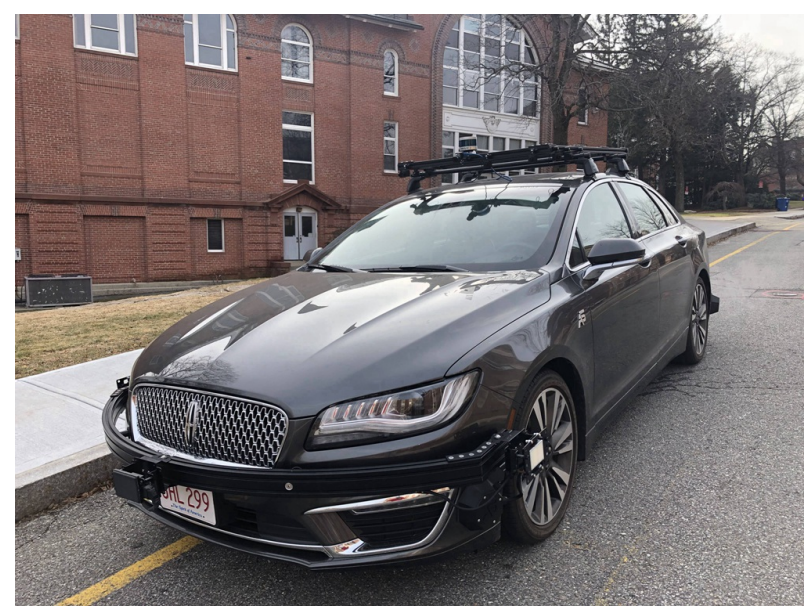

Fig. 1: A Lincoln MKZ equipped with 1 LiDAR, 1 front camera and 10 side cameras

LiDAR points when projected to the image plane. Thus calibration of the LiDAR-to-camera system needs to be improved.

In addition, LiDAR sensors have limited resolution according to their vertical axis, which makes it difficult to find correspondences between the points in a LiDAR frame and the exact pixels in the corresponding camera frame directly. Previous works have proposed several indirect methods to estimate the correspondences. Rangesh et al [14] and Geiger et al [3] assumed that the checkerboards have a flat surface and that all the LiDAR points on each checkerboard should be co-planar. However, traditional checkerboards made from cardboard, wood or aluminum are not flat enough for outdoor LiDAR-camera system calibration. To overcome this issue, [14] used a glass made calibration board to ensure nearperfect flatness and rigidity. On the other hand, Park et al [11] and Pereira et al [12] employed objects in special shapes to estimate the point-to-point correspondences. However, their works were also sensible to the lack of rigidity. Due to the same reasons, target-less approaches [6] also experienced large projection offsets. Moreover, due to the low vertical resolution, labeling correspondences for long-range calibration is difficult because the LiDAR sensor may not get enough lines of scans on the checkerboards or target objects. Thus few of the existing works can calibrate and validate their results for a distance longer than 5 meters.

In this paper, we introduce a new toolbox for LiDAR to camera calibration using a polygonal board. Through the automatic detection of the vertices in LiDAR frames and manual labeling the correspondence in camera frames, 


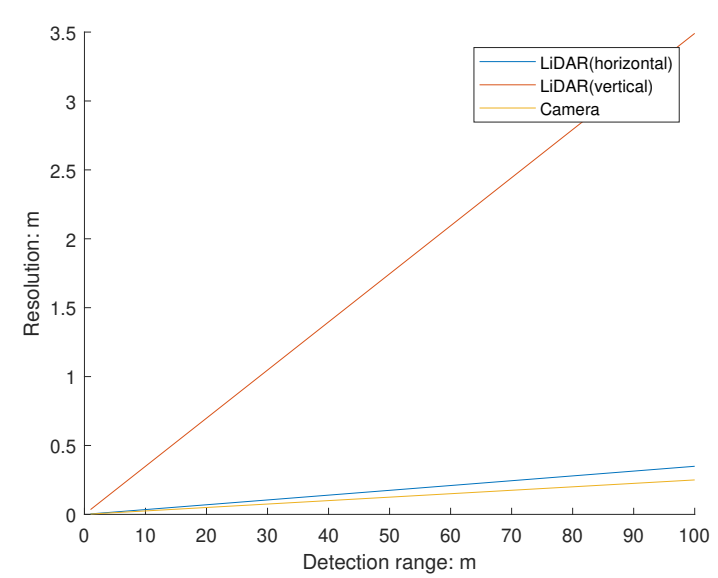

Fig. 2: Resolution of LiDAR and camera. The LiDAR has resolution of $0.2^{\circ}$ in vertical and $2^{\circ}$ in horizontal. The scale/focus ratio of the camera is 400 .

our solution collects direct point-to-point correspondences between LiDAR and camera coordinates. The correspondence pairs are used to estimate the intrinsic and extrinsic transforms via a genetic algorithm based approach. The rest of this paper is organized as follows: Section II discusses the related works. Section [II] describes the calibration models in the proposed method. In Section IV] we evaluate the proposed method on our LiDAR and Camera recordings. Section $\mathrm{V}$ gives the conclusions.

\section{RELATED WORK}

\section{A. LiDAR-camera correspondence collection}

Acquiring the LiDAR-camera correspondence is the first step of calibration. Since point-to-point correspondences are difficult to measure, two categories of indirect approaches were proposed in the literature.

one method is to estimate the point-to-point correspondences via knowledge of specific objects. [11] estimated the vertices of the rigid checkerboards in LiDAR frames by detecting the checkerboard edges. The approach of [12] detected the surface of the target ball and estimated the position of its center. This method relies on the clarity and precision of the target objects. They also assume that an intrinsic calibration of the camera is valid.

The other method does not rely on point-to-point correspondences. [3] and [14] assumed the LiDAR points on the checkerboard are coplanar and performed calibrations through planar-to-planar correspondences. Ishikawa et al [7] estimated the motion of LiDAR and camera separately and determined the intrinsics and extrinsics through motion-tomotion correspondences. Banerjee et al [1] detected the edges of objects in camera frames and calibrated through edge-to-edge correspondences. These approach s not sensitive to the quality of calibration equipment but relies on the segmentation of LiDAR and camera frames.

In this paper, we propose a point-to-point correspondence based approach. To overcome the limitations, we detect the vertices of target objects in a sequence of frames rather than a single frame. We also involve manual annotation of vertices in camera frames to increase the accuracy of correspondences.

\section{B. Solver of calibration}

Different optimizers have been used to estimate the parameters in the calibration models. Hulik et al [5] used 3D Hough transform to search for a continuous plane in point clouds. Velas et al [17] and Vasconcels et al [16] applied the RANSAC method to solve the extrinsic calibration. Paynot et al [13] and Zhang [20] introduced a probabilistic model to estimate the likelihood of a transformation and then iterated using the Levenberg-Marquardt algorithm. Heikkila et al [4] also used the Levenberg-Marquardt algorithm but applied direct linear transform (DLT) to solve the initial estimation of camera intrinsic parameters. Pandey et al [10] estimated the extrinsic calibration based upon the maximization of mutual information. In our work, a genetic algorithm is applied to estimate the parameters in extrinsic and intrinsic transforms since it works for non-linear models and avoids local optimal.

\section{Methodology}

This section gives an overview of our work on LiDAR to camera calibration. In this section, we first demonstrate the models applied in the proposed calibration, describe the data collection and processing, and then present the use of the genetic algorithm to solve the calibration task.

\section{A. Calibration models}

Models of LiDAR to camera projection have been well investigated. Suppose we have a scanned point $(x, y, z)$ in LiDAR coordinates, its corresponding point $(u, v, w)$ in camera coordinates, and its corresponding pint $(i, j)$ in the image plane. The transformation from LiDAR to the image includes two parts, as described in [4] [3].

The first one is the extrinsic transformation that is the projection model from LiDAR to camera coordinate. This 6-DOF matrix can be expressed as (1).

$$
\begin{gathered}
{\left[\begin{array}{l}
u \\
v \\
w
\end{array}\right]=\left[\begin{array}{ll}
\boldsymbol{R} & \boldsymbol{t} \\
\mathbf{0} & 1
\end{array}\right]\left[\begin{array}{l}
x \\
y \\
z \\
1
\end{array}\right]} \\
=\left[\begin{array}{cccc}
R_{11} & R_{12} & R_{13} & u_{0} \\
R_{21} & R_{22} & R_{23} & v_{0} \\
R_{31} & R_{32} & R_{33} & w_{0} \\
0 & 0 & 0 & 1
\end{array}\right]\left[\begin{array}{l}
x \\
y \\
z \\
1
\end{array}\right]
\end{gathered}
$$


where $R \in R^{3 \times 3}$ is the rotation matrix and $t \in R^{3 \times 1}$ is the translation vector. Since it is a linear projection and both coordinates share the same unit (meter), the rotation matrix $\mathrm{R}$ equals a multiplication of three sub-rotation matrix $R_{\text {roll }}$, $R_{\text {pitch }}$ and $R_{\text {yaw }}$, as shown in 2 .

$$
\begin{aligned}
& R=R_{\text {roll }} R_{\text {pitch }} R_{\text {yaw }} \\
& R_{\text {roll }}=\left[\begin{array}{ccc}
1 & 0 & 0 \\
0 & \cos (\alpha) & -\sin (\alpha) \\
0 & \sin (\alpha) & \cos (\alpha)
\end{array}\right] \\
& R_{\text {pitch }}=\left[\begin{array}{ccc}
\cos (\beta) & 0 & \sin (\beta) \\
0 & 1 & 0 \\
-\sin (\beta) & 0 & \cos (\beta)
\end{array}\right] \\
& R_{\text {yaw }}=\left[\begin{array}{ccc}
\cos (\gamma) & -\sin (\gamma) & 0 \\
\sin (\gamma) & \cos (\gamma) & 0 \\
0 & 0 & 1
\end{array}\right]
\end{aligned}
$$

Where $\alpha, \beta$, and $\gamma$ are the rotation angle along the $x, y$ and $z$ axis. The goal of the extrinsic matrix calibration is to estimate the 6 parameters $\left(\alpha, \beta, \gamma, u_{0}, v_{0}, w_{0}\right)$.

The second part is the intrinsic transformation that projects the $3 \mathrm{D}$ points in camera coordinate to the $2 \mathrm{D}$ image plane. Pinhole camera model and fisheye camera model are two popular intrinsic models. The pinhole model is described in (3).

$$
\left[\begin{array}{l}
i \\
j
\end{array}\right]=\left[\begin{array}{ccc}
f_{x} / w & 0 & i_{0} \\
0 & f_{y} / w & j_{0}
\end{array}\right]\left[\begin{array}{l}
u \\
v \\
1
\end{array}\right]
$$

Where $f_{x}$ and $f_{y}$ are the focus lengths of the lens system along $\mathrm{x}$ and $\mathrm{y}$ axis, and $i_{0}, j_{0}$ are the offsets on the target image plane. For the fisheye model, lens distortion and tangential distortion and skew are also considerated as in (4).

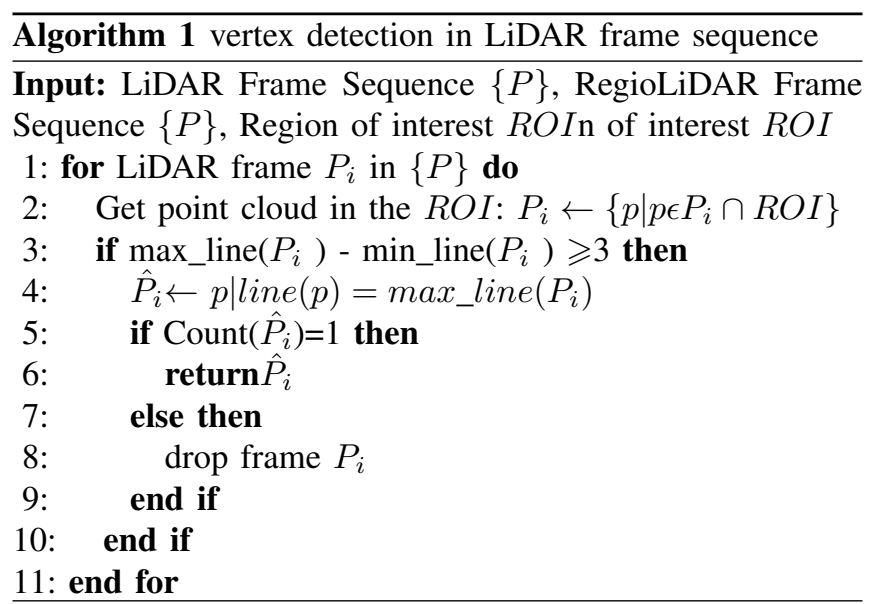

$$
\begin{gathered}
x_{d}=\left(1+k_{1} r^{2}+k_{2} r^{4}+k_{5} r^{6}\right)\left[\begin{array}{c}
u / w \\
v / w
\end{array}\right] \\
d x=\left[\begin{array}{c}
2 k_{3} u v+k_{4}\left(r^{2}+2 u^{2}\right) \\
k_{3}\left(r^{2}+2 v^{2}\right)+2 k_{4} u v
\end{array}\right] \\
{\left[\begin{array}{c}
i \\
j
\end{array}\right]=\left[\begin{array}{ccc}
f_{x} & \alpha_{c} \cdot f_{x} & i_{0} \\
0 & f_{y} & j_{0}
\end{array}\right]\left[\begin{array}{c}
u \\
v \\
1
\end{array}\right]}
\end{gathered}
$$

Recentprogressinautomat

Where $r=\sqrt{u^{2}+v^{2}}$.

In general, an intrinsic transformation using pinhole model has 4 parameters $\left(f_{x}, f_{y}, i_{0}, j_{0}\right)$ and the one using fisheye has 10 parameters $\left(f_{x}, f_{y}, i_{0}, j_{0}, \alpha_{c}, k_{1}, k_{2}, k_{3}, k_{4}, k_{5}\right)$.

\section{B. data collection and processing}

The key issue of point-to-point based approaches in LiDAR to camera calibration is to collect the point-to-point pairs between LiDAR and camera coordinates. Since LiDAR point cloud is sparse on the vertical axis, it is difficult to get the target correspondence on a checkerboard in a single frame. In our approach, we detect the vertices of the checkerboard in a sequence of LiDAR frames.

The proposed approach has the following assumptions: (1) only the checkerboard and the tester holding the checkerboard exist in the region of interest (ROI), and (2) the target vertex of the checkerboard is the highest point in the ROI. Comparing to other related works, our approach does not require a perfect checkerboard nor a considerable number of scans of the checkerboards. The proposed approach not only reduces the difficulty of calibration task but also makes it capable of calibrating for low-resolution LiDAR devices such as 4-line LiDAR and 16-line LiDAR. The procedures to detect the vertex in a LiDAR frame sequence are described in Algorithm 1 . 


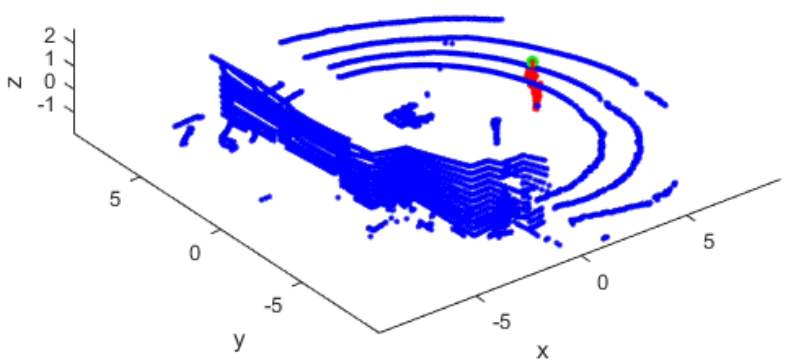

(a)

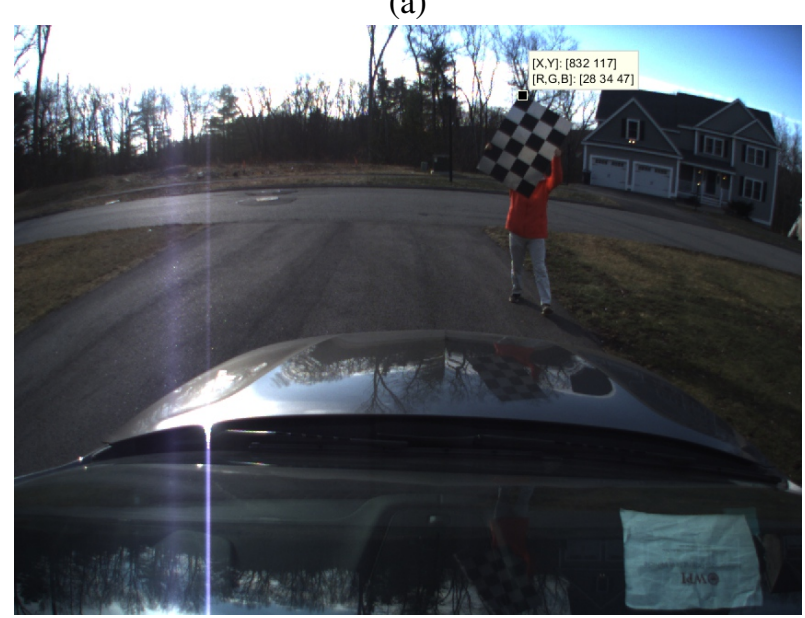

(b)

Fig. 3: Example LiDAR frame (a) and example camera frame (b)

When the toolbox starts, it shows a LiDAR frame and the user needs to input the ROI threshold. For the subsequent LiDAR frames, the toolbox segments the points inside the ROI, validates the size of segmented object and searches for the top line inside the ROI. If the top line has only one point, it is stored as a vertex points, otherwise the LiDAR frame is dropped. For each frame in which the vertex is detected, the toolbox asks the user's confirmation and presents the corresponding camera frame for annotation if a valid vertex is detected. In practice, the LiDAR and camera sensors might not be perfectly synchronized, so the toolbox select the camera frame that is captured nearly at the same time of the LiDAR scan. After each annotation, the toolbox gets a new point-to-point correspondence between the LiDAR and camera coordinate.

\section{Genetic Algorithm}

The proposed toolbox calibrates the extrinsic and intrinsic transformations of LiDAR to camera through the genetic algorithm (GA). GA is a widely used algorithm in parameter estimation since it is model-based, data-driven and robust to non-linear optimization. In theory, a GA instance rolls out thousands of alternative parameter sets and selects certain candidates with least offset as seeds of the next generation. The algorithm terminates when determined generations are

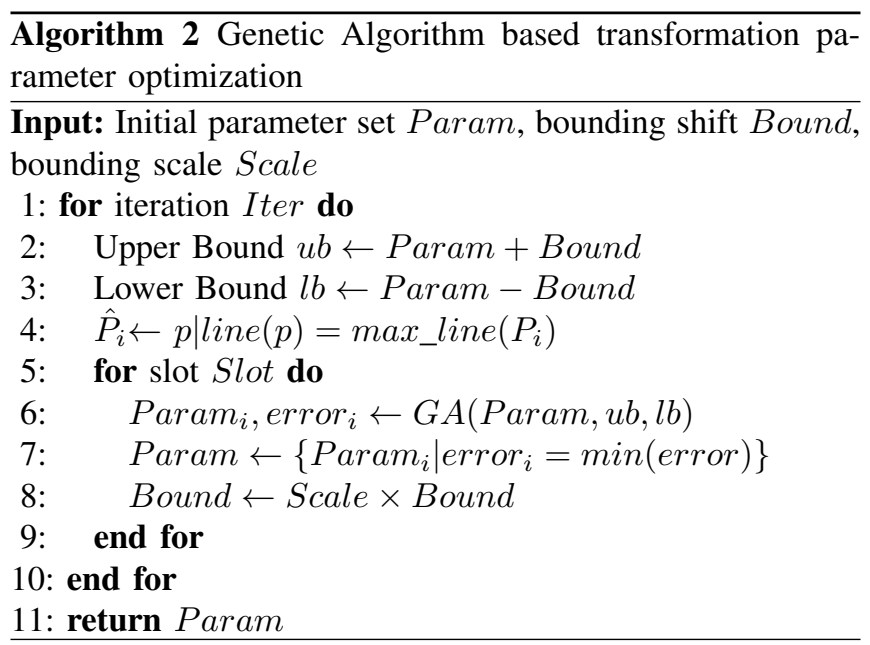

reached or the convergence goal is reached. Comparing to gradient-based algorithms, GA is capable to avoid local optima.

In this toolbox, we employe the MATLAB genetic algorithm toolbox as the GA solver. According to the calibration model, we set 10 parameters for pinhole-model based solution and 16 parameters for fisheye-model based solution. The average offset of labeled points projected from LiDAR to the image plane is selected as the target loss of GA. The GA process is iterated multiple times until the loss does not decrease. In each iteration, the toolbox initializes 5 GA slots to avoid local optima. Each GA slot keeps an 800 population and runs 30 generations to optimize the parameters. In the first iteration, the upper bound and lower bound of GA slots are manually determined. During the rest of the iterations, the toolbox narrows the upper bound and lower bound according to the optima of last iteration and the number of iterations it has executed. The iteration algorithm is described in Algorithm 2 .

\section{EXPERIMENT}

In the experiment, we use the proposed toolbox to calibrate the LiDAR-camera system of our autonomous vehicle prototype. The system as shown in Figure 4 contains a Velodyne VLP-16 LiDAR and a Point Grey Chameleon 3 camera. Both devices are installed on the roof rack facing the front. The LiDAR has 16 row scanners covering a $\left[-15^{\circ}, 15^{\circ}\right]$ vertical field of view and $360^{\circ}$ horizontal one resulting in a $0.2^{\circ}$ horizontal resolution and a $2^{\circ}$ vertical resolution. The camera is adjusted to $15^{\circ}$ looking down to the ground.

To calibrate this LiDAR-camera system, we use a $2 \times 3$ feet checkerboard rotated $45^{\circ}$ so that one vertex is up. A person holds the checkerboard and moves around in a $10 \mathrm{~m} \times 30 \mathrm{~m}$ field in front of the car. We recorded the data sequence in about 219 seconds that contains 11582 camera frames and 7639 LiDAR frames. After processing the data, 222 LiDAR-to-camera correspondences are collected as shown in Figure 5. The lower bound and upper bound setting is manually determined as shown in Table I based on the device setting and installation. For validation, we record another 


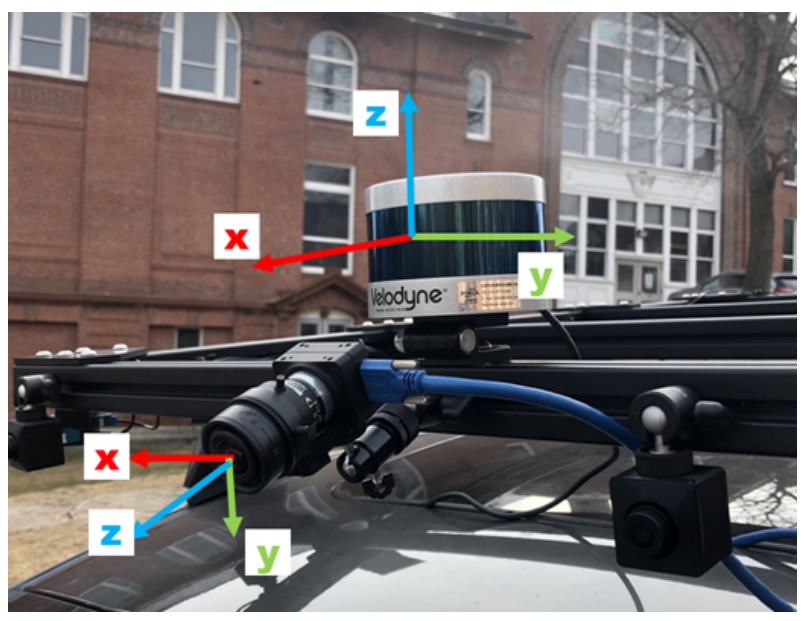

Fig. 4: LiDAR-camera system on an Lincoln MKZ

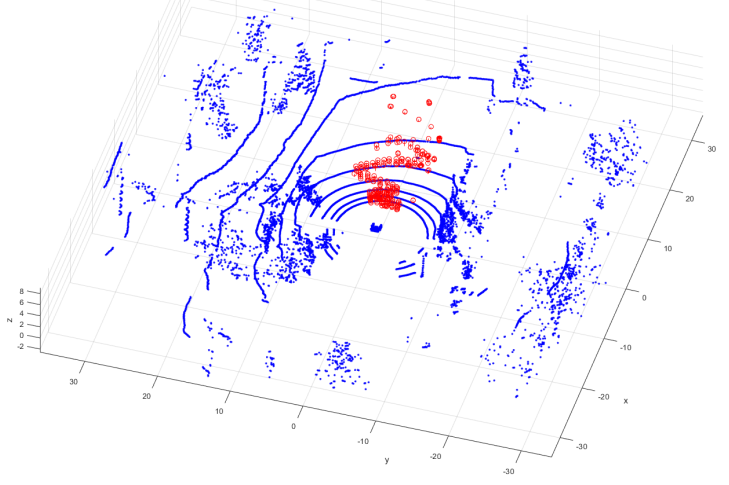

Fig. 5: Labeled data in LiDAR cooridate frame. The blue dots are the point cloud from one LiDAR frame, and the stems present the position of labeled correspondences in the LiDAR coordinate.

data sequence with 3293 camera frames and 2177 LiDAR frames, and annotated 358 correspondences. For the training data, this calibration method is able to achieve 6.35 pixel offset in average for pinhole camera model and 5.02 pixel for fisheye camera model. On the test data, it results 6.75 pixel error in average using pinhole camera model and 5.1 pixel using fisheye camera model. An example projection result is shown in Figure 6 The result shows that the proposed approach generates precise transformation from LiDAR to camera coordinate. Figure 7 and 8 shows the validation of the LiDAR-camera transformation along the LiDAR coordinate. It is obvious that the offset around the camera normal is small, but as the angle to the camera normal grows, the offset grows to as much as 16 pixels, which indicates the current camera intrinsic models may not be precise enough.

For further testing, we drive the test vehicle on the road and record a 1043-frame LiDAR and camera sequence. From the data, we detect the lane markers by thresholding the intensity of LiDAR points in the lowest ring. We then project the detected lane markers to the corresponding camera frames using the calibrated transformation function and

\begin{tabular}{|c|c|c|c|c|c|}
\hline parameter & $\alpha$ & $\beta$ & $\gamma$ & $u_{0}$ & $v_{0}$ \\
\hline lower boundary & $0.2 \pi$ & $-0.8 \pi$ & $-0.3 \pi$ & -1 & -1 \\
\hline upper boundary & $0.8 \pi$ & $-0.2 \pi$ & $0.3 \pi$ & +1 & +1 \\
\hline parameter & $w_{0}$ & $f_{x}$ & $f_{y}$ & $i_{0}$ & $j_{0}$ \\
\hline lower boundary & -1 & 300 & 300 & 300 & 300 \\
\hline upper boundary & +1 & 900 & 900 & 900 & 900 \\
\hline parameter & $k_{1}$ & $k_{2}$ & $k_{3}$ & $k_{4}$ & $k_{5}$ \\
\hline lower boundary & -1 & -1 & -1 & -1 & -1 \\
\hline upper boundary & +1 & +1 & +1 & +1 & +1 \\
\hline
\end{tabular}

TABLE I: Boundaries of parameters for GA solver

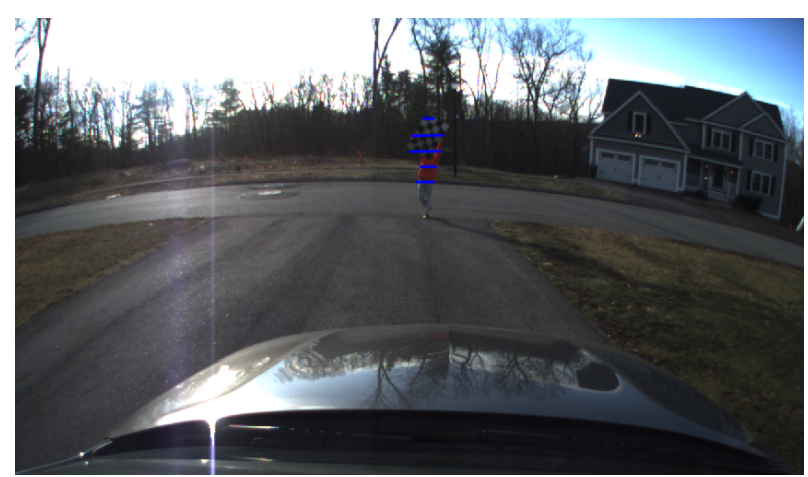

Fig. 6: Example calibration result

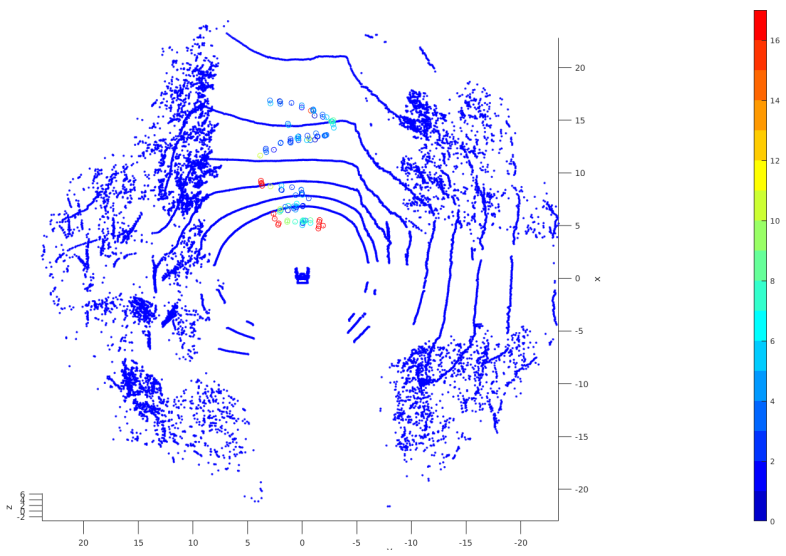

Fig. 7: Validation of LiDAR-camera calibration using pinhole camera model

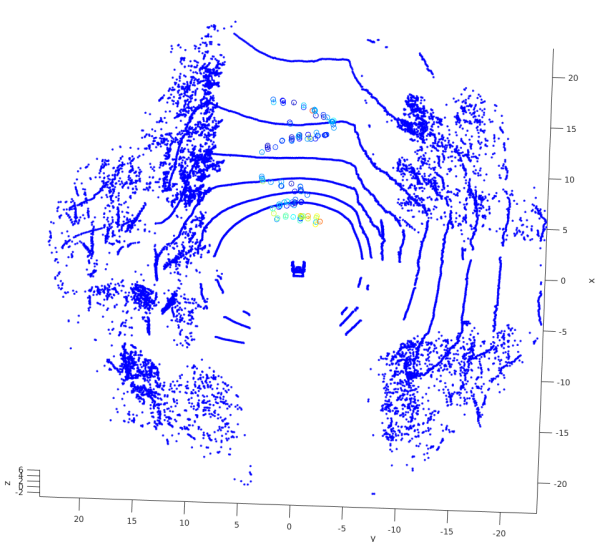

Fig. 8: Validation of LiDAR-camera calibration using fisheye camera model 


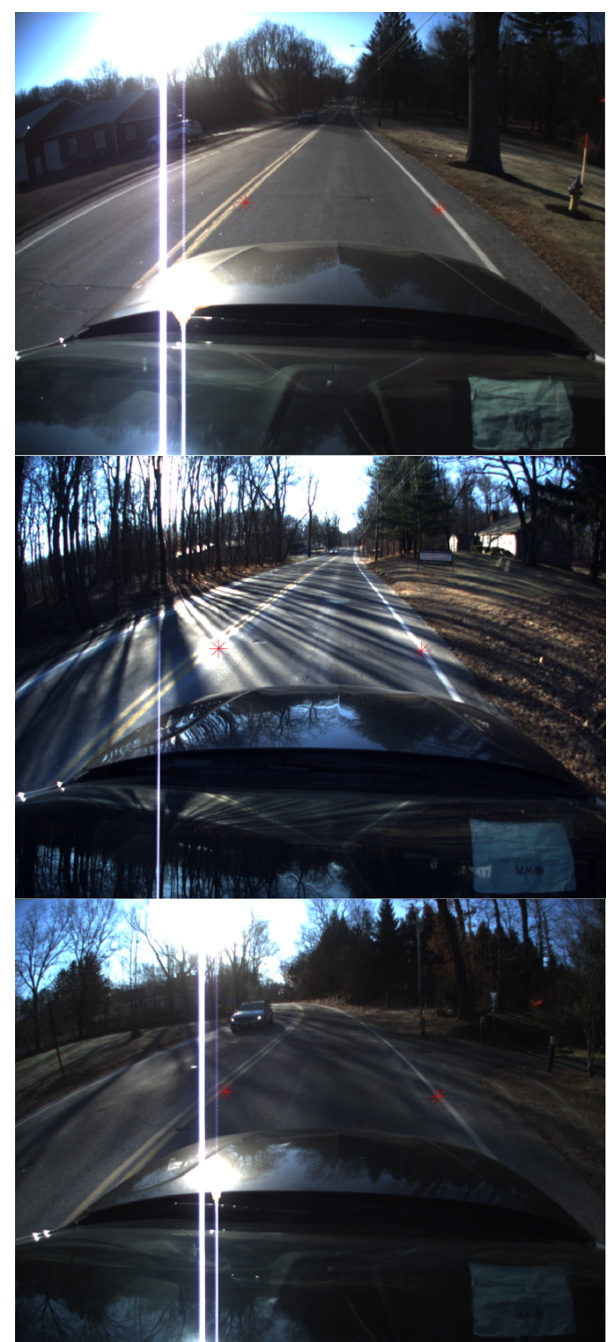

Fig. 9: Lane markers detected in LiDAR frames and projected to Camera frames

visualize them on the camera frames with red asterisks. As shown in Figure 9 the offset of the lane markers is acceptable for automated driving.

\section{Conclusions}

In this paper, we propose a novel and accurate approach for LiDAR-to-camera system calibration. This approach is not sensitive to the checkerboard quality and works for long-range calibration. We also develop a MATLAB based toolbox to calibrate the LiDAR and camera. The toolbox automatically detects the vertex in a LiDAR frame sequence and provide a convenient user interface to annotate the correspondence in camera frames. A genetic algorithm based approach is applied to estimate the extrinsic and intrinsic parameters. Experiment on test vehicle shows that the toolbox works well on long range calibration and achieves good results.

Future work will investigate automatic corner detection on camera frames, extend the calibration to LiDAR-thermal camera systems, and investigate the correspondences of $2 \mathrm{D}$ to $3 \mathrm{D}$ projection.

\section{REFERENCES}

[1] Koyel Banerjee, Dominik Notz, Johannes Windelen, Sumanth Gavarraju, and Mingkang He. Online Camera LiDAR Fusion and Object Detection on Hybrid Data for Autonomous Driving. 2018 IEEE Intelligent Vehicles Symposium (IV), (Iv):1632-1638, 2018.

[2] Xiaozhi Chen, Huimin Ma, Ji Wan, Bo Li, and Tian Xia. Multi-view $3 \mathrm{~d}$ object detection network for autonomous driving. In IEEE CVPR, volume 1, page 3, 2017.

[3] Andreas Geiger, Frank Moosmann, Ömer Car, and Bernhard Schuster. Automatic camera and range sensor calibration using a single shot. Proceedings - IEEE International Conference on Robotics and Automation, pages 3936-3943, 2012.

[4] Janne Heikkila, Olli Silven, and Infotech Oulu. A Four-step Camera Calibration Procedure with Implicit Image Correction. pages 11061112, 1997.

[5] Rostislav Hulik, Michal Spanel, Pavel Smrz, and Zdenek Materna. Continuous plane detection in point-cloud data based on 3D Hough Transform. 2014

[6] Kiyoshi Irie, Masashi Sugiyama, and Masahiro Tomono. Targetless camera-LiDAR extrinsic calibration using a bagged dependence estimator. IEEE International Conference on Automation Science and Engineering, 2016-Novem:1340-1347, 2016.

[7] Ryoichi Ishikawa, Takeshi Oishi, and Katsushi Ikeuchi. LiDAR and Camera Calibration using Motion Estimated by Sensor Fusion Odometry. apr 2018.

[8] Y. Lyu, L. Bai, and X. Huang. Real-time road segmentation using lidar data processing on an fpga. In 2018 IEEE International Symposium on Circuits and Systems (ISCAS), pages 1-5, May 2018.

[9] Yecheng Lyu, Lin Bai, and Xinming Huang. Chipnet: Real-time lidar processing for drivable region segmentation on an fpga. IEEE Transactions on Circuits and Systems I: Regular Papers, 2018.

[10] Gaurav Pandey, James R. McBride, Silvio Savarese, and Ryan M. Eustice. Automatic Extrinsic Calibration of Vision and Lidar by Maximizing Mutual Information. Journal of Field Robotics, 2015.

[11] Yoonsu Park, Seokmin Yun, Chee Sun Won, Kyungeun Cho, Kyhyun Um, and Sungdae Sim. Calibration between color camera and 3D LIDAR instruments with a polygonal planar board. Sensors (Switzerland), 2014.

[12] Marcelo Pereira, David Silva, Vitor Santos, and Paulo Dias. Self calibration of multiple LIDARs and cameras on autonomous vehicles. Robotics and Autonomous Systems, 2016.

[13] Thierry Peynot and Abdallah Kassir. Laser-Camera Data Discrepancies and Reliable Perception in Outdoor Robotics. 2010.

[14] Akshay Rangesh, Kevan Yuen, Ravi Kumar Satzoda, Rakesh Nattoji Rajaram, Pujitha Gunaratne, and Mohan M. Trivedi. A Multimodal, Full-Surround Vehicular Testbed for Naturalistic Studies and Benchmarking: Design, Calibration and Deployment. sep 2017.

[15] Tixiao Shan and Brendan Englot. Lego-loam: Lightweight and groundoptimized lidar odometry and mapping on variable terrain. 2018.

[16] Francisco Vasconcelos, Joao P Barreto, and Urbano Nunes. A Minimal Solution for the Extrinsic Calibration of a Camera and a LaserRangefinder. Technical report.

[17] Martin Velas, Michal Spanel, Zdenek Materna, and Adam Herout. Calibration of RGB Camera With Velodyne LiDAR.

[18] Yan Yan, Yuxing Mao, and Bo Li. Second: Sparsely embedded convolutional detection. Sensors, 18(10):3337, 2018.

[19] Ji Zhang and Sanjiv Singh. Loam: Lidar odometry and mapping in real-time. In Robotics: Science and Systems, volume 2, page 9, 2014.

[20] Zhengyou Zhang. A Flexible New Technique for Camera Calibration; a typo in Appendix B) (last updated on Aug A Flexible New Technique for Camera Calibration. Technical report, 2008. 